\title{
Oncolytic virotherapy with human telomerase reverse transcriptase promoter regulation enhances cytotoxic effects against gastric cancer
}

\author{
TOMOYA KATO $^{1}$, MIKIHITO NAKAMORI ${ }^{1}$, SHUICHI MATSUMURA ${ }^{1}$, MASAKI NAKAMURA $^{1}$, \\ TOSHIYASU OJIMA ${ }^{1}$, HIROSHI FUKUHARA ${ }^{2}$, YASUSHI INO ${ }^{3}$, TOMOKI TODO ${ }^{3}$ and HIROKI YAMAUE ${ }^{1}$ \\ ${ }^{1}$ Second Department of Surgery, Wakayama Medical University, School of Medicine, Wakayama 641-8510; \\ ${ }^{2}$ Department of Urology, Kyorin University, School of Medicine, Mitaka, Tokyo 181-8611; \\ ${ }^{3}$ Division of Innovative Cancer Therapy, The Advanced Clinical Research Center, \\ The Institute of Medical Science, The University of Tokyo, Tokyo 108-8639, Japan
}

Received October 19, 2020; Accepted March 18, 2021

DOI: $10.3892 / \mathrm{ol} .2021 .12751$

\begin{abstract}
Currently, gastric cancer is the third most common cause of cancer-associated mortality worldwide. Oncolytic virotherapy using herpes simplex virus (HSV) has emerged as a novel therapeutic strategy against cancer. Telomerase is activated in $>90$ of malignant tumors, including gastric cancer, and human telomerase reverse transcriptase (hTERT) is one of the major components of telomerase enzyme. Therefore, in oncolytic HSV, placing the essential genes under the regulation of the hTERT promoter may enhance its antitumor efficacy. The present study examined the antitumor effect of fourth-generation oncolytic HSVs, which contain the ICP6 gene under the regulation of the hTERT promoter (T-hTERT). To examine the association between hTERT expression and prognosis in patients with gastric cancer, immunohistochemical analysis of resected tumor specimens was performed. The enhanced efficacy of T-hTERT was determined in human gastric cancer cell lines in vitro and in human gastric adenocarcinoma specimens in vivo. In in vitro experiments, enhanced cytotoxicity of T-hTERT was observed in MKN1, MKN28 and MKN45 cells compared with that of a third-generation oncolytic HSV, T-null. In particular, the cytotoxicity of T-hTERT was markedly enhanced in MKN45 cells. Furthermore, in vivo experiments demonstrated that 36.7 and $54.9 \%$ of cells were found to be lysed $48 \mathrm{~h}$ after infection with T-null or T-hTERT viruses at $0.01 \mathrm{pfu} / \mathrm{cell}$, respectively. The T-hTERT-treated group exhibited considerably lower cell
\end{abstract}

Correspondence to: Dr Mikihito Nakamori, Second Department of Surgery, Wakayama Medical University, School of Medicine, 811-1 Kimiidera, Wakayama 641-8510, Japan

E-mail: chamcham@wakayama-med.ac.jp

Key words: gastric cancer, oncolytic virotherapy, herpes simplex virus, human telomerase reverse transcriptase promoter, oncolytic virus viability than the control [phosphate-buffered saline (-)] group. Therefore, employing oncolytic HSVs that contain the ICP6 gene under the regulation of the hTERT promoter may be an effective therapeutic strategy for gastric cancer. To the best of our knowledge, the present study was the first to describe the effect of an oncolytic HSV with ICP6 expression regulated by the hTERT promoter on gastric cancer cells.

\section{Introduction}

Gastric cancer is currently the fifth most common cancer and the third most common cause of cancer-related mortality worldwide $(1,2)$. Although there have been advancements in early diagnosis and therapeutic management through surgery, chemotherapy, and molecular targeted drugs, the long-term prognosis of patients with metastasis or recurrence remains poor (3). Based on these findings, novel therapeutic strategies for advanced and recurrent gastric cancers are needed.

As a promising novel approach for cancer therapy, oncolytic viruses have recently emerged as a cancer treatment because of their specific properties (4). Oncolytic virotherapy is a therapeutic strategy that uses replication-competent viruses to infect and destroy cancer cells (5). The key desirable characteristics of any oncolytic virus are as follows: Specificity for the targeted cancer, the potency to kill infected cancer cells, cross-prime antitumor immunity, and low toxicity to avoid adverse reactions and prevent pathogenic reversion (6). Various oncolytic viruses with these characteristics, such as adenovirus, herpes simplex virus (HSV), vaccinia virus, and reovirus are currently undergoing preclinical or clinical studies.

In particular, oncolytic HSVs were engineered to target tumor tissues for selective replication and amplification at the tumor site with minimal replication in normal tissues, thereby resulting in efficient clearance and reduced toxicity. Compared with other oncolytic viruses that have been investigated for oncolytic purposes, HSVs possess unique features. Hence, many oncolytic HSVs have been developed and modified for cancer therapy (7). Most of them have been engineered to delete the neuronal toxicity gene ICP34.5 in order to 
target tumor tissues for selective replication and amplification. Other strategies have focused on eliminating important genes necessary for viral replication. ICP6 encodes a crucial enzyme for nucleotide metabolism and viral DNA synthesis in non-dividing cells. G207, a mutant HSV type 1 with deleted ICP6, was the first to be engineered (8). Another strategy for achieving tumor-specific HSV type 1 replication using tumor-specific promoters such as the survivin promoter (9), hypoxia-inducible factor responsive promoter (10), and the probasin-derived promoter $\mathrm{ARR}_{2} \mathrm{~PB}$ has been reported (11). Although these viruses preferentially replicate in tumor cells to activate each tumor-specific promoter, these oncolytic HSVs target only a subset of specific tumor types. In addition, for safety concerns, antiherpetic drug medications such as acyclovir and ganciclovir are available to overcome undesired infection or toxicity caused by the HSV. Several clinical studies using oncolytic HSV mutants (1716, G207, and NV1020) have been conducted (12-14). Talimogene laherparepvec showed therapeutic benefit against melanoma in a phase III clinical trial (15) and was approved by the US Food and Drug Administration for the treatment of melanoma in 2015.

In preclinical studies, several oncolytic HSV mutants showed an antitumor effect on gastric cancer cells (16-18). Previously, we demonstrated that an oncolytic virus with thrombospondin-1 (TSP-1) enhanced the efficacy of oncolytic HSVs in gastric cancer cells, and the combination of TSP-1 and oncolytic HSVs inhibited the cancer cell proliferation both in vitro and in vivo (19). In this experimental study, however, oncolytic HSVs expressing TSP-1 did not show cytotoxicity to all types of gastric cancer cell lines, and some cell lines were resistant to treatment. Collectively, a newly designed oncolytic HSV treatment based on the biological properties of viruses and gastric cancer cells is needed.

Therefore, we hypothesized that the antitumor effects can be enhanced by regulating the expression of ICP6, a gene crucial for viral replication, with a tumor-specific promoter such as the human telomerase reverse transcriptase (hTERT) promoter, which has demonstrated promising results $(20,21)$ since telomeres play an important role in maintaining cellular homeostasis and senescence $(22,23)$. As DNA polymerase fails to fully synthesize DNA termini, human telomeres in somatic cells undergo progressive shortening with cell division (24). Many studies have already demonstrated that telomerase is activated in more than $90 \%$ of malignant tumors but is strictly repressed in normal somatic cells (25-27). Therefore, using the hTERT promoter to regulate the replication of this critical oncolytic virus may increase tumor selectivity and lead to enhanced antitumor potency.

In this study, we employed the hTERT promoter to regulate the expression of ICP6 present in the oncolytic HSV genome. The antitumor effect of oncolytic HSV containing ICP6 gene under the regulation of the hTERT promoter was investigated not only in gastric cancer cell lines but also in freshly resected gastric cancer specimens. This modified oncolytic HSV showed enhanced antitumor effects both in vitro and in vivo.

\section{Materials and methods}

Patients and histological analysis. Tumor samples of International Union Against Cancer stage II or III were collected from overall 45 patients who underwent curative resection for gastric cancer at Wakayama Medical University Hospital from January 2010 to December 2010. The diagnostic procedure for gastric cancer fulfilled the following criteria included for analyses in the current study: Patients with primary gastric cancer with preoperatively diagnosed by endoscopy; patients who were not administered chemotherapy before surgery; and patients with no signs of ascites, distant metastases, or bulky para-aortic lymph node metastases after physical examination and enhanced CT scan evaluation. They included 26 stage II and 19 stage III gastric cancer patients based on Tumor Node Metastasis (TNM) Classification of the International Union Against Cancer (28). The mean age of the patients was 71.3 years, and there were 30 male and 15 female subjects. The follow-up period was five years. Stage II and III patients based on TNM classification without submucosal cancer received S-1 (oral fluoropyrimidine)-based postoperative adjuvant chemotherapy. The present study was approved by the Human Ethics Review Committee of Wakayama Medical University (approval no. 1657). Informed consent was obtained in the form of opt-out on the web page of Wakayama Medical University from all patients in accordance with the guidelines of the Ethical Committee on Human Research of our institution.

Immunohistochemical analysis of hTERT expression was performed using an anti-telomerase reverse transcriptase mouse monoclonal antibody (sc-393013; Santa Cruz Biotechnology, Inc.) as described previously (29). Pretreatment was performed by autoclaving the tissues in citrate buffer (pH 6.0) for $7 \mathrm{~min}$ at $121^{\circ} \mathrm{C}$. Endogenous peroxidase activity was blocked with $3 \%$ hydrogen peroxide in methanol. Nonspecific binding sites were blocked with $0.25 \%$ casein in phosphate-buffered saline (PBS) containing stabilizing protein and $0.015 \mathrm{~mol} / \mathrm{l}$ sodium azide. Primary antibodies were diluted in PBS, then added to the samples followed by overnight incubation at $4^{\circ} \mathrm{C}$. Following two washes, the sections were incubated for $90 \mathrm{~min}$ at room temperature with Histofine Simple Stain MAX-PO (MULTI) (Nichirei). Finally, the reaction products were stained with a 3,3'-diaminobenzidine substrate, counterstained with hematoxylin, dehydrated with ethanol, and fixed with xylene.

Evaluation of immunohistochemistry. For scoring assessments, the cells were counted in five separate areas of intratumoral regions under x400 high-power magnification. The staining intensity was defined as follows: 0 , no staining; $1+$, weak; $2+$, moderate; and 3+, strong (Fig. 1A). The predominant intensity was chosen in case of areas with different staining intensities. The quantification of positivity (0-100\%) was based on an estimate of the percentage of stained cancer cells in the lesion. The final immunostaining scores were calculated by multiplying the staining intensity with the percentage of positive cells, thereby generating immunostaining scores ranging from 0 to 300 (30-32). The cutoff values of the immunostaining scores were set as the median value, as per previous reports (33-35).

Cell lines. Vero (African green monkey kidney normal cell line), MKN1, MKN28, MKN45, MKN74, NUGC3, NUGC4, KATOIII, and N87 (human gastric cancer cell lines) cells were 
A

0

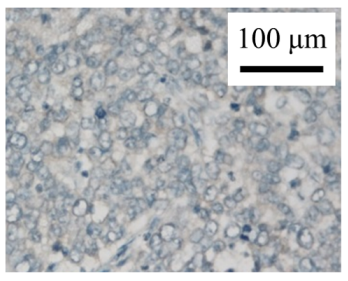

$1+$

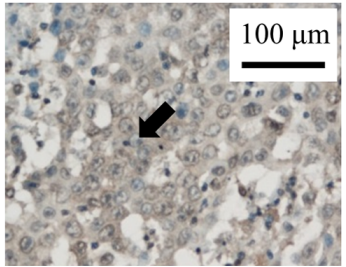

$2+$

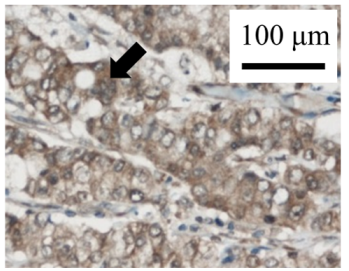

$3+$

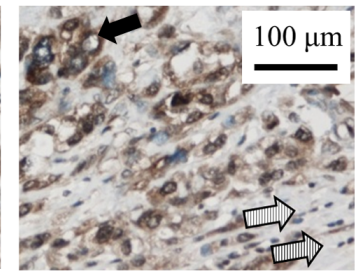

B

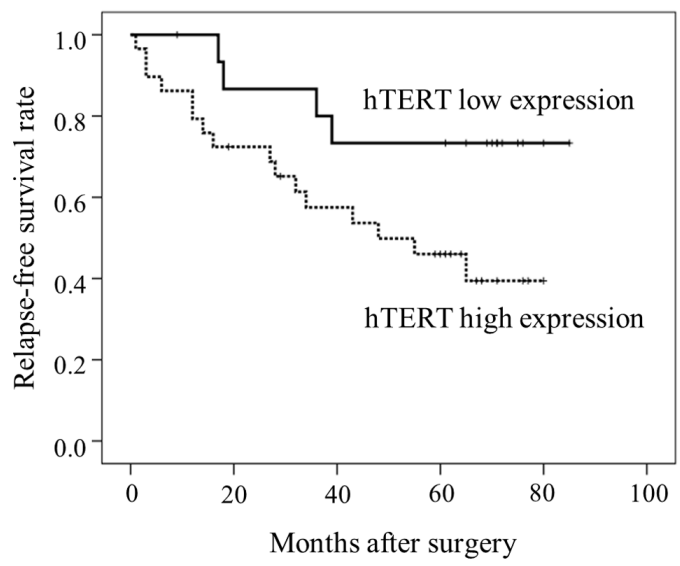

C

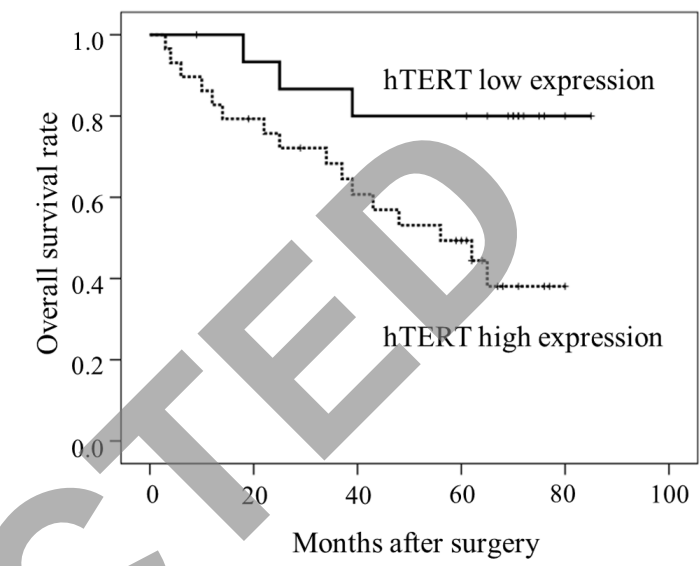

Figure 1. Immunohistochemical staining of hTERT and the association between hTERT expression intensity and prognosis. (A) Immunohistochemical staining of hTERT. Image of staining intensity grade: (0) no staining, $(1+)$ weak, $(2+)$ moderate, and (3+) strong intensity. hTERT was expressed only in cancer cells (indicated by thick arrows) and not in normal cells (indicated by hatched arrows). hTERT in gastric cancer samples was stained and observed mainly in the cytoplasm. (B) Relapse-free survival of 45 patients who underwent radical resection and were stratified using the median level according to hTERT expression. A significant difference was observed between the hTERT high expression group and the low expression group (P=0.048; log-rank test). (C) Overall survival of 45 patients who underwent radical resection and were stratified according to hTERT expression. A significant difference was observed between the hTERT high expression group and the low expression group $(\mathrm{P}=0.020$, log-rank test). hTERT, human telomerase reverse transcriptase.

obtained from the RIKEN BioResource Center. All cell lines were authenticated according to the Cell Line Verification Test Recommendations of ATCC Technical Bulletin no. 8 (2008). TMK-1 cells, a human gastric cancer cell line, were provided by Dr Eiichi Tahara (Hiroshima University, Hiroshima, Japan). All human gastric cancer cell lines were cultured in RPMI-1640 and Vero cells were cultured in Dulbecco's modified Eagle's medium both supplemented with $10 \%$ fetal bovine serum (Thermo Fisher Scientific, Inc.).

Genomic structure of the virus. T-hTERT is a fourth-generation oncolytic HSV, which was provided to us by Dr Tomoki Todo (The University of Tokyo, Tokyo, Japan). It was constructed by deleting the $\alpha 47$ gene and both copies of the $\gamma 34.5$ gene, with the hTERT promoter regulating the ICP6 gene expression (Fig. 2A). $\gamma 34.5$ is a major determinant of HSV neurovirulence and blocks host shutoff of protein synthesis in response to viral infection. Lack of this function is likely responsible for the less efficient growth of $\gamma 34.5$-mutants when compared with wild-type HSV, as observed in many tumor cell types. This double mutation confers important advantages such as minimal chance of reverting to wild-type, preferential replication in tumor cells, attenuated neurovirulence, and ganciclovir and acyclovir hypersensitivity (36). Because of the overlapping transcripts encoding ICP47 and US11, the deletion in $a 47$ also places the late US11 gene under the control of the immediate-early $a 47$ promoter. This alteration in US11 expression enhances the growth of g34.5-mutants by preventing protein synthesis shutoff (36). ICP6 encodes a large subunit of ribonucleotide reductase (RR), an enzyme critical for nucleotide metabolism and viral DNA synthesis in non-dividing cells but not in dividing cells. By placing the ICP6 gene under the hTERT promoter, the hTERT promoter is activated in tumor cells and expresses ICP6. T-null is an HSV-1-based oncolytic virus, constructed by deleting ICP6, $\alpha 47$, and both copies of $\gamma 34.5$ and are not regulated by the hTERT promoter (Fig. 2A). Viral stocks were prepared by releasing the virus from infected Vero cells with heparin followed by high-speed centrifugation, as described previously (37).

In vitro cytotoxicity of T-null in gastric cancer cell lines. T-null was used to treat gastric cancer cell lines in vitro. The cells were seeded on 6 -well plates at a density of $5 \times 10^{5}$ cells/well and incubated. Following a 24-h incubation, the cells were infected with T-null at $0.1 \mathrm{pfu} / \mathrm{cell}$ for $1 \mathrm{~h}$ and further incubated at $37^{\circ} \mathrm{C}$. Cells were collected at 24 or $48 \mathrm{~h}$ after infection and stained with trypan blue, and the number of viable cells was counted. The survival rate was expressed as the percentage of the PBS (-)-treated control cells.

Comparison of T-null and T-hTERT cytotoxicity in gastric cancer cell lines. For virus yield studies, MKN1 and MKN45 cells, which are minimally sensitive to T-null, and MKN28 
A

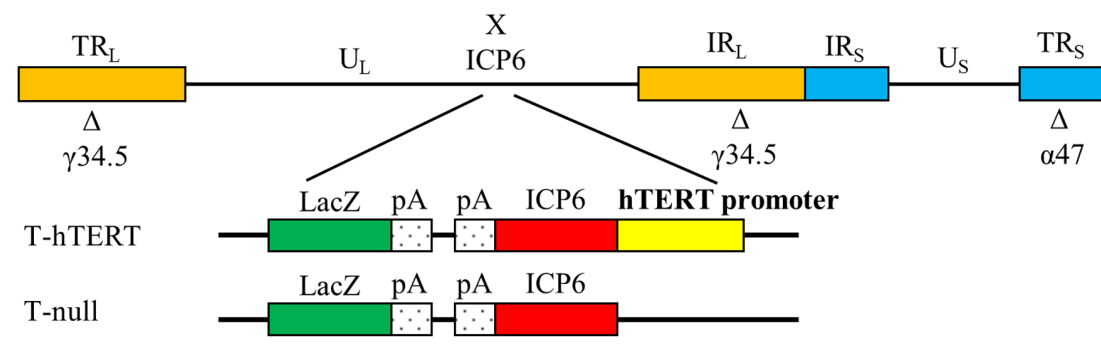

B

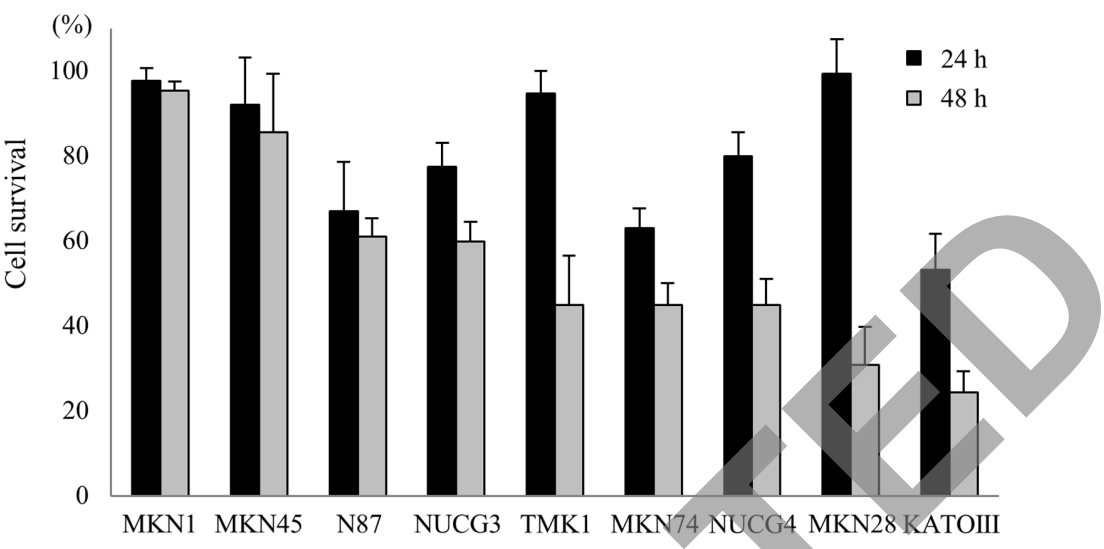

$\mathrm{C}$

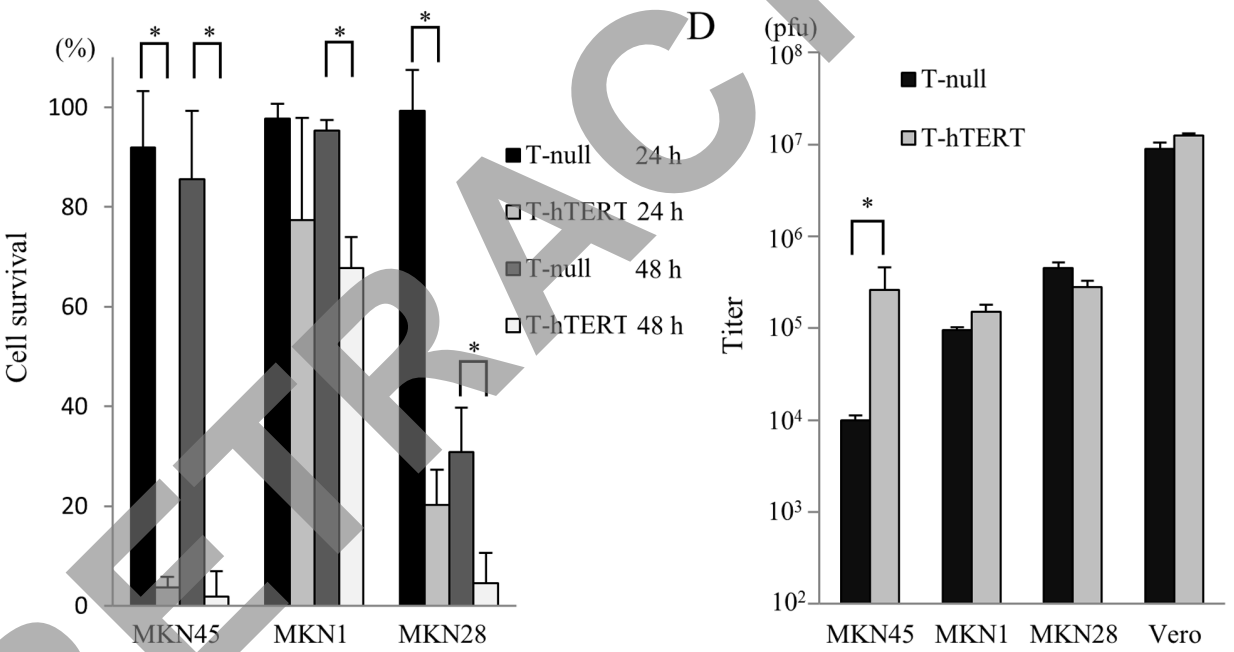

Figure 2. Genomic structure of the oncolytic virus, and in vitro cytotoxicity and viral replication of oncolytic herpes simplex viruses in gastric cancer cell lines. (A) T-hTERT was constructed by deleting the $\alpha 47$ gene and both copies of the $\gamma 34.5$ gene, and by placing the ICP6 gene under regulation by the hTERT promoter. T-null was constructed by deleting the ICP6 gene, $\alpha 47$ gene and both copies of the $\gamma 34.5$ gene and is not under the control of the hTERT promoter. (B) In vitro cytotoxicity of T-null in gastric cancer cell lines. The number of surviving cells is expressed as a percentage of the PBS (-)-treated control. (C) Comparison of T-null and T-hTERT cytotoxicity in MKN45, MKN1 and MKN28 cells. The number of surviving cells is expressed as a percentage of the PBS (-)-treated control. "P<0.01. (D) Comparison of viral replication between T-null and T-hTERT in MKN45, MKN1, MKN28 and Vero cells. The viral titer of T-hTERT was $\sim 10$-fold higher than that of T-null in MKN45 cells. "P<0.01. hTERT, human telomerase reverse transcriptase; ICP6, a gene encodes a viral ribonucleotide reductase; T-hTERT, oncolytic HSVs which contain the ICP6 gene under the regulation of the hTERT promoter; T-null, oncolytic HSVs which contain the ICP6 gene not regulated by the hTERT promoter.

cells, which are moderately sensitive to T-null, were seeded on 6 -well plates at a density of $5 \times 10^{5}$ cells/well and incubated for $24 \mathrm{~h}$. Each well was infected with either T-null or T-hTERT at $0.1 \mathrm{pfu} / \mathrm{cell}$ for $1 \mathrm{~h}$ and further incubated at $37^{\circ} \mathrm{C}$. After 24 or $48 \mathrm{~h}$ of incubation, the cells were collected and stained with trypan blue, and the number of viable cells was counted. The survival rate was expressed as the percentage of the PBS (-)-treated control cells.

In vitro replication assay. MKN1, MKN28, MKN45, and Vero cells were seeded on 6 -well plates at a density of $5 \times 10^{5}$ cells/well and incubated for $24 \mathrm{~h}$. Each well was infected with either
T-null or T-hTERT at $0.01 \mathrm{pfu} / \mathrm{cell}$ for $1 \mathrm{~h}$ and further incubated at $37^{\circ} \mathrm{C}$. After a $24-\mathrm{h}$ incubation, the cells were scraped and lysed by repeating the process of freezing and thawing three times. Titration of the progeny virus was measured on Vero cells via plaque assays. Each experiment was performed in triplicates.

Western blot analysis. Gastric cancer cell lines were seeded in $100 \mathrm{~mm}$ dishes at a density of $1 \times 10^{6}$ cells/dish and incubated at $37^{\circ} \mathrm{C}$. After a $24-\mathrm{h}$ incubation, the cells were treated with PBS (-), T-null, or T-hTERT at $0.01 \mathrm{pfu} /$ cell for $1 \mathrm{~h}$, incubated further at $37^{\circ} \mathrm{C}$ for $24 \mathrm{~h}$, and harvested. Western blot analysis was performed as described previously (38). Anti-RRM2 
antibody (sc-10846; Santa Cruz Biotechnology, Inc.) or goat polyclonal anti- $\beta$-actin antibody (sc-47778, Santa Cruz Biotechnology, Inc.) were used as primary antibodies (39). Goat IgG HRP-conjugated antibody was used as the secondary antibody (HAF017, R\&D Systems).

Ex vivo assessment of oncolytic HSV cytotoxicity in gastric cancer. As described in a previous report (40), surgical sections of cancer tissue were collected and incubated in collagen medium for a short time (within $72 \mathrm{~h}$ ) to evaluate the antitumor effect of oncolytic HSV. In this experiment, gastric cancer samples were collected at Wakayama Medical University Hospital from October 2016 to December 2016. They included 2 stage II and 4 stage III gastric cancer patients based on TNM Classification of the International Union Against Cancer. The mean age was 74.3 years (65-85) with 5 males and 1 female subjects. We carried out all experiments in compliance with the Declaration of Helsinki, the guidelines for ethical principles for medical research involving human subjects, and the ethical guidelines of Wakayama Medical University. This ex vivo study was also approved by the Committee of Animal Experiments and Gene Recombination (approval no. 26-31) of Wakayama Medical University. Informed consent was obtained in the written form from all patients in accordance with the guidelines of the Ethical Committee on Human Research of our institution. In general, human gastric cancer specimens collected through radical gastrectomy were incubated ex vivo on collagen gel immediately after resection. Cellmatrix type I-A collagen (Nitta Gelatin), reconstitution buffer [2.2\% (w/v) $\mathrm{NaHCO}_{3}$ $0.2 \mathrm{M}$ HEPES, and $50 \mathrm{mM} \mathrm{NaOH}$ ], and 10X RPMI-1640 medium were mixed at a ratio of 8:1:1 and poured into 24-well dishes $\left(0.5 \mathrm{ml} /\right.$ well). Tissues were cut into $2 \mathrm{~mm}^{3}$ pieces and placed on collagen gel. Each well was treated with PBS (-), T-null, or T-hTERT at $0.01 \mathrm{pfu} / \mathrm{cell}$ for $1 \mathrm{~h}$ and incubated at $37^{\circ} \mathrm{C}$ for $48 \mathrm{~h}$. The cell viability of gastric cancer tissues was assessed using a CellTiter 96 AQueous One Solution Cell Proliferation Assay (Promega Corp.) as per the manufacturer's instructions.

Statistical analysis. Comparison of categorial variables between hTERT expression and clinicopathological characteristics of gastric cancer patients was analyzed using the unpaired Student's t-test (for age and tumor size) and a Fisher's exact test (for sex, histological type, macroscopic type and pathological TNM classification). Multivariate analysis of overall survival was used the Cox proportional hazards regression model. The survival data were analyzed by the Kaplan-Meier method and the log-rank (Mantel-Cox) test. Quantitative data are reported as means \pm standard deviation. For comparison between two groups, significant differences were determined using the unpaired Student's t-test. For comparison of multiple groups, statistical significance was determined with a one-way ANOVA followed by a Tukey's post hoc test. All analyses were performed using the SPSS statistics version 21 software (IBM Corp.). P-values $<0.05$ were considered statistically significant.

\section{Results}

Relationship between survival rate and hTERT in gastric cancer. Immunohistochemical analyses were performed on paraffin-embedded tissues collected from 45 patients with gastric cancer. For immunohistochemistry, hTERT in gastric cancer samples was stained and observed mainly in the cytoplasm (Fig. 1A). hTERT was expressed only in cancer cells and not in normal cells (Fig. 1A). The hTERT expression scores were calculated for each sample. The median score of hTERT was 100 (range, 0-300). The binarization of the score data for this marker present in a high expression group $(\mathrm{n}=29)$ versus a low expression group $(\mathrm{n}=16)$ at the median level was performed. Patient clinicopathological characteristics are listed in Table I.

Kaplan-Meier survival curves showed the overall survival of patients with gastric cancer, characterized based on the results of hTERT expression analysis. The survival curves of the 45 patients who underwent curative $\mathrm{R} 0$ resection revealed a significantly poorer relapse-free survival rate in the hTERT high expression group than in the low expression group $(\mathrm{P}=0.048$; Fig. 1B). Moreover, a significantly poorer prognosis was observed in the hTERT high expression group than in the low expression group $(\mathrm{P}=0,029$; Fig. $1 \mathrm{C})$.

Multivariate overall survival analysis was calculated using the Cox proportional hazard regression model. Multivariate analysis revealed that hTERT expression was an independent prognostic factor in patients with gastric cancer $(\mathrm{P}=0.031$, hazard ratio=3.918; Table II).

In vitro cytotoxicity of T-null in gastric cancer cell lines. After $48 \mathrm{~h}$ of infection with T-null at $0.1 \mathrm{pfu} / \mathrm{cell}, 39.0 \%$ of N87, $40.1 \%$ of NUGC3, 55.0\% of TMK-1, 55.0\% of MKN74, $55.1 \%$ of NUGC4, $69.1 \%$ of MKN28, and $75.6 \%$ of KATOIII cells were lysed. However, only $4.7 \%$ of MKN1 and $14.4 \%$ of MKN45 cells were lysed by T-null infection (Fig. 2B). Therefore, these results suggested that the sensitivity to T-null virus varies among human gastric cancer cell lines. Therefore, we examined the cytotoxicity of T-hTERT or T-null in MKN28 cells, which are moderately sensitive gastric cancer cell lines, and MKN45 and MKN1, which are minimally sensitive gastric cancer cell lines.

Comparing the cytotoxicity of T-null and T-hTERT in gastric cancer cell lines. After $48 \mathrm{~h}$ of infection with oncolytic HSVs at $0.1 \mathrm{pfu} / \mathrm{cell}$, the cytotoxicity of T-hTERT was found to be more than that of T-null in all cell lines. Particularly in MKN45 cells, T-hTERT showed increased cytotoxicity as compared with T-null (Fig. 2C). Therefore, we further examined the differences in viral replication between T-hTERT- and T-null-infected cells.

In vitro replication assay. We compared the replication potencies of T-hTERT with those of T-null in MKN45, MKN1, MKN28, and Vero cells. The results showed that the viral titer of T-hTERT was approximately 10-fold higher than that of T-null in MKN45 cells $(\mathrm{P}<0.01)$. However, in MKN1 and MKN28 cells, the titers of T-hTERT were not remarkable different from those of T-null (Fig. 2D).

Expression of RRM2 in gastric cancer cell lines. According to our preliminary studies, we confirmed that telomerase activity and expression of hTERT mRNA expressed in human gastric cancer cell lines (Figs. S1 and S2). RR plays 
Table I. Clinicopathological characteristics.

\begin{tabular}{lllr}
\hline Characteristic & $\begin{array}{c}\text { hTERT high expression } \\
\text { group }(\mathrm{n}=29)\end{array}$ & $\begin{array}{c}\text { hTERT low expression } \\
\text { group }(\mathrm{n}=16)\end{array}$ & P-value \\
\hline Sex, $\mathrm{n}($ male/female) & $19 / 10$ & $11 / 5$ & 0.548 \\
Median age, years (range) & $71.0(39-86)$ & $69.5(48-86)$ & 0.837 \\
Median tumor size, mm (range) & $45(12-150)$ & $41(20-140)$ & 0.403 \\
Macroscopic type, $\mathrm{n}(0 / 1 / 2 / 3 / 4)$ & $2 / 3 / 10 / 11 / 3$ & $0 / 1 / 7 / 7 / 1$ & 0.770 \\
Histological type, $\mathrm{n}$ & $12 / 17$ & $6 / 10$ & 0.528 \\
differentiated/undifferentiated) $_{\mathrm{T}, \mathrm{n}(1 \mathrm{a} / 1 \mathrm{~b} / 2 / 3 / 4 \mathrm{a} / 4 \mathrm{~b})^{\mathrm{a}}}$ & $0 / 2 / 8 / 7 / 8 / 4$ & $0 / 0 / 4 / 7 / 5 / 0$ & 0.324 \\
$\mathrm{~N}, \mathrm{n}(0 / 1 / 2 / 3)^{\mathrm{a}}$ & $5 / 9 / 8 / 4 / 3$ & $3 / 7 / 3 / 2 / 1$ & 0.907 \\
$\mathrm{M}, \mathrm{n}(0 / 1)^{\mathrm{a}}$ & $29 / 0$ & $16 / 0$ & $>0.999$ \\
Stage, $\mathrm{n}(\mathrm{IIA} / \mathrm{IIB} / \mathrm{IIIA} / \mathrm{IIIB} / \mathrm{IIIC})^{\mathrm{a}}$ & $6 / 9 / 5 / 5 / 4$ & $2 / 9 / 2 / 2 / 1$ & 0.583 \\
\hline
\end{tabular}

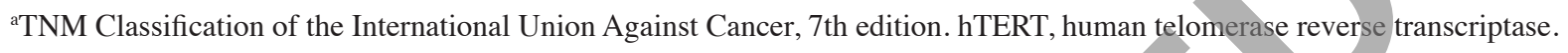

Table II. Multivariate analysis of overall survival of 45 patients with gastric cancer.

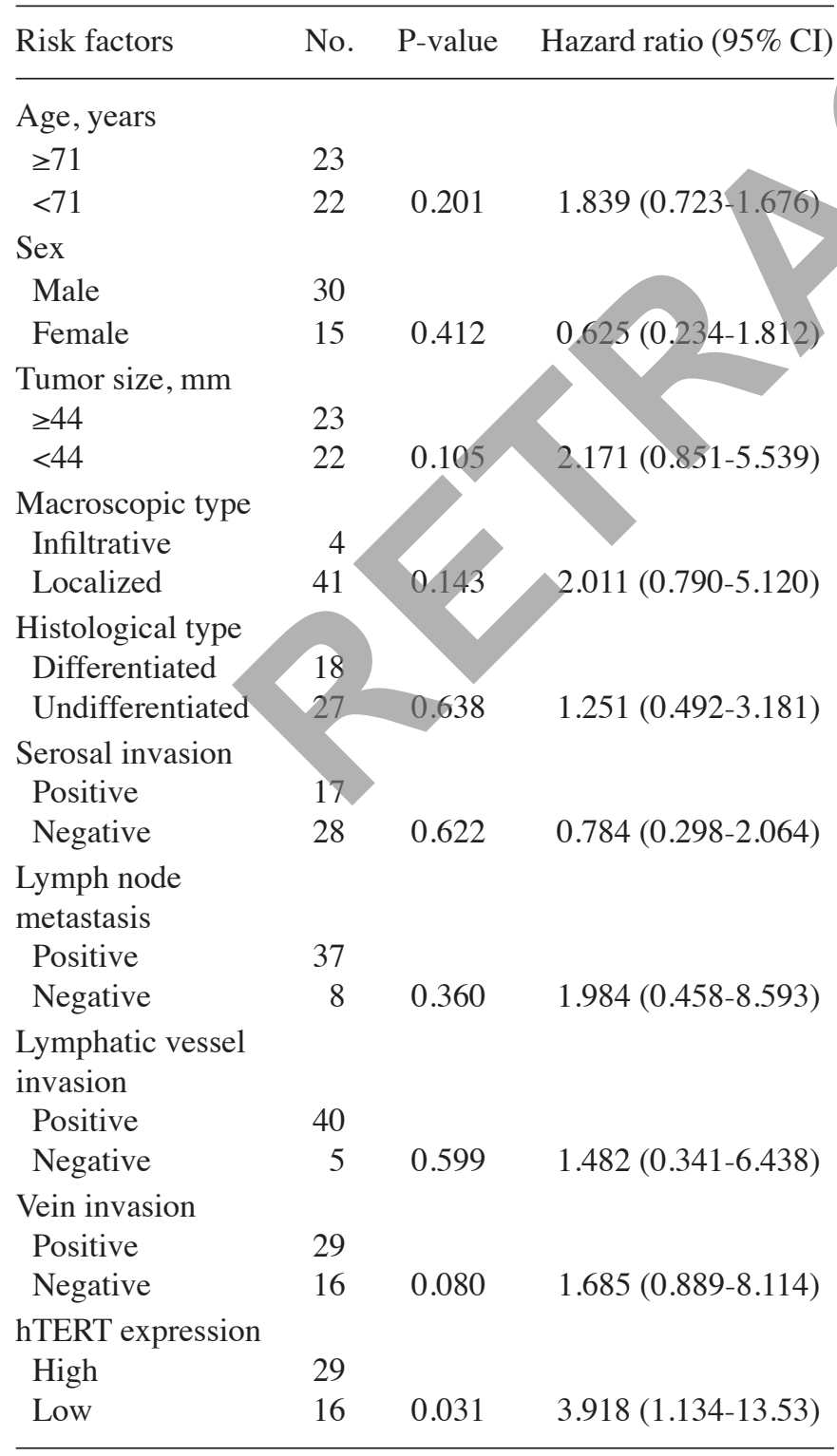

CI, confidence interval; hTERT, human telomerase reverse transcriptase. an essential role in converting ribonucleoside diphosphate to 2-deoxyribonucleoside diphosphate to maintain the homeostasis of nucleotide pools (41). Human RR consists of two subunits, M1 and M2 (42). RR enzymatic activity is modulated by the expression of its M2 (RRM2) subunit (43). Therefore, we carried out a western blot analysis to examine RRM2 protein expression in the gastric cancer cell lines. Although almost all the cell lines expressed RRM2, its expression in MKN45 cells was very low (Fig. 3A). Additionally, MKN45 cells infected with T-null showed very low expression of RRM2, whereas those infected with T-hTERT showed high expression of RRM2 and its expression levels were almost 5-fold higher than those infected with T-null (Fig. 3B and C).

Ex vivo assessment of oncolytic HSV cytotoxicity in gastric cancer. In terms of experimental animal protection and management, an important part of our present study was the analysis of clinical cancerous samples freshly obtained from patients with gastric cancer. As it is accepted that established cell lines differ from the initial clinical tumors, it was not completely unexpected to see a different profile of viral transfer in comparison with the cell lines. To clarify that T-hTERT remains unaffected with the heterogeneity of gastric cancer tissue, collagen gel culture consisting of gastric cancer clinical tissue samples was synthesized. To examine the effects of oncolytic HSVs in gastric cancer in vivo, human gastric adenocarcinoma specimens collected through radical gastrectomy were incubated on collagen gel immediately after resection and treated with PBS (-), T-null, or T-hTERT. After $48 \mathrm{~h}$ of incubation, these specimens were subjected to frozen sectioning and further examined by hematoxylin and eosin staining. In the gastric cancer specimens with oncolytic HSV infection, lysis was observed in the tumor cells (Fig. 4A and B). After $48 \mathrm{~h}$ of infection with T-null or T-hTERT at $0.01 \mathrm{pfu} / \mathrm{cell}$, 36.7 and $54.9 \%$ of cells were lysed by the viruses, respectively. The T-hTERT-infected group showed significantly lower cell viability than that of the control [PBS (-)] group $(\mathrm{P}=0.029$; Fig. 4C). However, as compared with T-null, T-hTERT did not show any significant cytotoxic effects $(\mathrm{P}=0.37$; Fig. $4 \mathrm{C})$. 
A

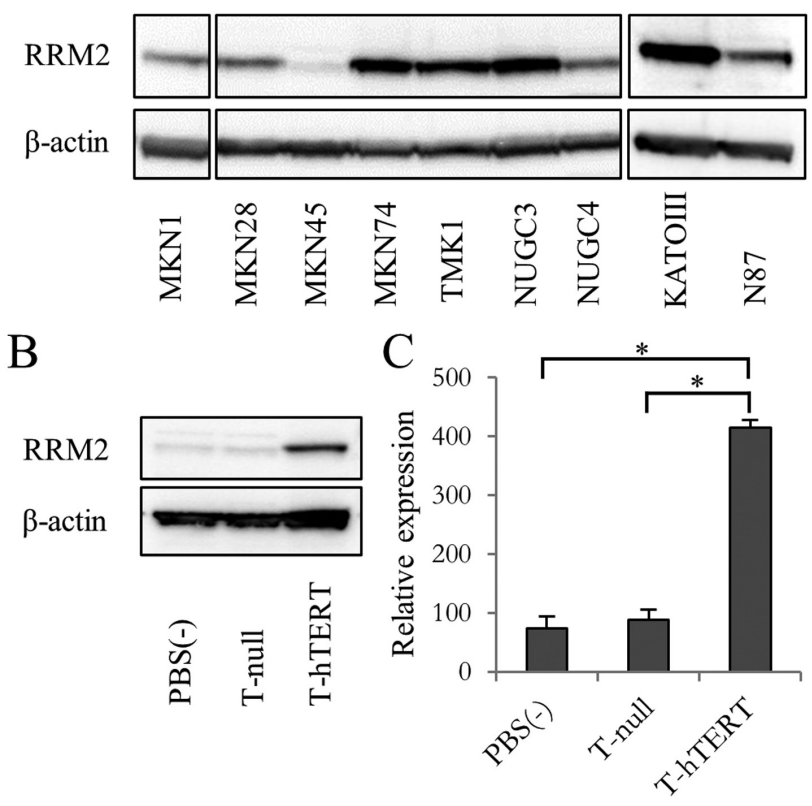

A

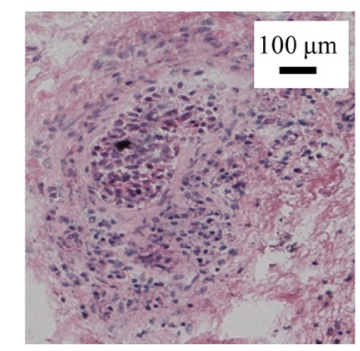

B

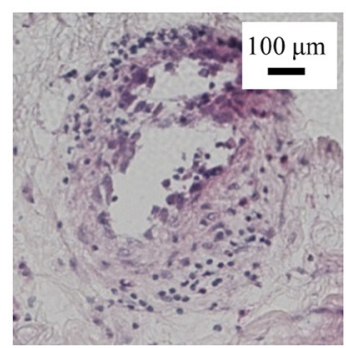

$\mathrm{C}$

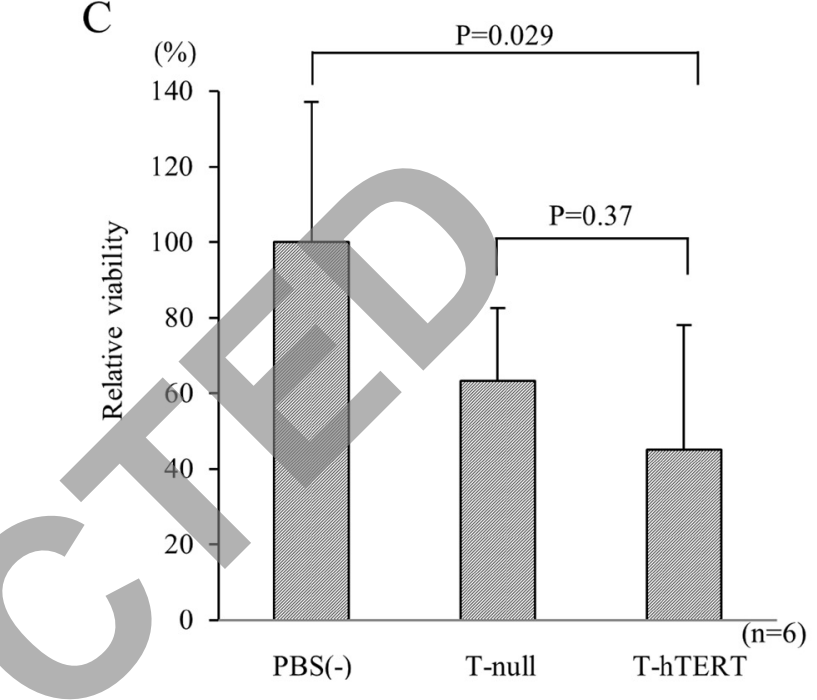

Figure 3. Expression levels of RRM2 subunit in gastric cancer cell lines. (A) Expression levels of the RRM2 subunit were examined by western blotting. Each group of samples was measured separately under the same exposure conditions. Almost all cell lines expressed RRM2; however RRM2 expression was very low in MKN45 cells. (B) Expression levels of RRM2 were examined in MKN45 cells infected with T-hTERT. MKN45 cells were infected with PBS (-) (left), T-null (middle) or T-hTERT (right). (C) Expression levels of RRM2 in MKN45 cells were semi-quantified using a densitometer and RRM2 expression was increased almost 5-fold compared with the T-null group. " $\mathrm{P}<0.005$. RRM2, ribonucleotide reductase M2, hTERT, human telomerase reverse transcriptase; T-hTERT, oncolytic HSVs which contain the ICP6 gene under the regulation of the hTERT promoter; T-null, oncolytic HSVs which contain the ICP6 gene not regulated by the hTERT promoter.

\section{Discussion}

Figure 4. Ex vivo assessment of oncolytic herpes simplex virus cytotoxicity in gastric cancer. (A) Gastric cancer specimens treated with PBS (-). (B) Gastric cancer specimens treated with oncolytic herpes simplex viruses. In tumor cells, cell lysis was observed. (C) At $48 \mathrm{~h}$ after infection with T-null or T-hTERT at 0.01 pfu/cell, 36.7 and $54.9 \%$ of cells had been killed, respectively. The T-hTERT treatment group exhibited significantly lower cell viability compared with the control [PBS $(-)$ ] group $(\mathrm{P}=0.029)$. hTERT, human telomerase reverse transcriptase; T-hTERT, oncolytic HSVs which contain the ICP6 gene under the regulation of the hTERT promoter; T-null, oncolytic HSVs which contain the ICP6 gene not regulated by the hTERT promoter.

The main goal of this study was to develop a newly designed oncolytic HSV-based treatment by using the biological properties of viruses and gastric cancer cells. The viral ICP6 gene of HSV encodes the large subunit of RR, which generates sufficient deoxynucleotide 5'-triphosphate pools for efficient viral DNA replication $(7,8)$, and is abundantly expressed in cancer cells but not in non-dividing cells. Although many variants of oncolytic HSVs containing the ICP6 gene have been developed (8-11), the expression of RR in these viruses is not regulated by tumor-specific promoters. In this study, we first described the impact of an oncolytic HSV with ICP6 expression regulated by the hTERT promoter on gastric cancer cells. It was previously reported that hTERT expression is observed in most cancer cells, and almost no hTERT expression is observed in non-cancerous cells (44-47). Moreover, a telomerase-specific oncolytic adenovirus, OBP-301, was found to considerably reduce tumor weight and increase survival in a nude mouse model of gastric cancer (48-50).

We evaluated the feasibility of using an hTERT promoter to regulate oncolytic $\mathrm{HSV}-1$ replication and described its antitumor effect on gastric cancer cell lines. Preliminary clinical specimens were used to clarify the relationship

between hTERT expression and prognosis. hTERT expression was observed in 43 of 45 patients (95\%), in accordance with the previous studies reporting that hTERT is expressed in most tumor types (43-46). In addition, 29 of 45 patients (64\%) showed high hTERT expression, and their prognosis was poorer than that for patients with low hTERT expression. It was revealed that hTERT expression was correlated with prognosis in stage II or III gastric cancer patients requiring postoperative adjuvant chemotherapy. Based on this correlation, we hypothesized that regulating the replication of oncolytic HSV, a telomerase-dependent oncolytic virus, by the hTERT promoter, could be useful for gastric cancer treatment. G47 $\Delta$ is a triple-mutated, third-generation oncolytic HSV-1, which was developed by introducing another deletion mutation to the genome of a second-generation oncolytic HSV-1, G207 (51,52). Therefore, in our study, we generated a newly designed oncolytic HSV with ICP6 gene regulated by the hTERT promoter, which has the same genetic backbone as $\mathrm{G} 47 \Delta$.

Additionally, we compared the oncolytic activity of T-hTERT and T-null, in which ICP6 expression is not 
regulated by the hTERT promoter. The data showed that T-hTERT generally exhibited similar oncolytic activity to that of T-null in cancer cell lines potentially expressing RR. Furthermore, even in cell lines with low RR expression, T-hTERT showed a stronger antitumor effect than T-null. In these experiments, the effect of the viruses was assessed only on cell lines and thus, the efficacy of the treatment in patients may be different as it is well-known that established cell lines differ from the primary tumors from which they were derived (53). Therefore, we evaluated the antitumor effect of T-hTERT in clinical samples.

For this purpose, we compared the oncolytic activity of T-hTERT and T-null in freshly resected gastric cancer specimens. T-hTERT showed a notable antitumor effect stronger than that exhibited by T-null. In addition, these oncolytic HSVs lysed tumor cells but not normal cells, which was pathologically confirmed. These results suggest that T-hTERT has tumor specificity, which is an essential factor for oncolytic virus therapy, and is, therefore, a promising and pivotal oncolytic agent.

In contrast, several limitations of this study should be acknowledged. Recently, immune checkpoint blockade has attracted attention and has demonstrated excellent treatment results in some cancers, including gastric cancer. In addition to tumor lysis, oncolytic viruses can induce host immune responses against cancer cells. The success of checkpoint inhibitors has indicated that enhancing antitumor immunity can be effective. In fact, in clinical trials, the combination of oncolytic herpes virotherapy and immune checkpoint blockade has proven to be an effective treatment for melanoma patients (54). At present, combinatorial therapy using T-hTERT and immune checkpoint blockade for gastric cancer may achieve enhanced antitumor effects. Hence, further studies are needed to investigate this possibility.

Collectively, in conclusion, this study is the first to report oncolytic HSV therapy for human gastric cancer by using viruses in which ICP6 expression is regulated by the hTERT promoter. We showed that hTERT regulation enhanced the efficacy of oncolytic HSV in gastric cancer cells and inhibited cell proliferation both in vitro and in vivo. Our data suggested that ICP6 expression controlled by the hTERT promoter enhances HSV replication and induces cytotoxicity in gastric cancer cells. Further studies are needed to determine whether the antitumor immunity stimulated by T-hTERT treatment can facilitate the antitumor effect of T-hTERT. Clinical trials are, therefore, required to verify these findings.

\section{Acknowledgements}

Not applicable.

\section{Funding}

This work was supported by Japan Society for the Promotion of Science (JSPS) KAKENHI (Grant-in-Aid for Scientific Research) (grant nos. 23591946, 26461992 and 17k10604), and in part by grants from Practical Research for Innovative Cancer Control, Japan Agency for Medical Research and Development (AMED) (grant nos. 19ck0106416h0002 and 17 ck0106144h0003)

\section{Availability of data and materials}

The datasets used and/or analyzed during the current study are available from the corresponding author on reasonable request.

\section{Authors' contributions}

TK, MiN, TT and HY conceived the current study. TK, MiN and MaN conceived and designed the experiments. TK and SM performed the experiments. TK, MiN and HY confirmed the authenticity of all the raw data. HF and YI contributed to acquisition of data. TK, SM and TO contributed to statistical analysis and interpretation of the data. TK was involved in all stages of the study and performed the immunohistochemical staining. TK and SM were major contributors in writing the manuscript. MiN and HY reviewed and revised the manuscript. All authors drafted and edited the manuscript. All authors read and approved the final manuscript.

\section{Ethics approval and consent to participate}

All gastric cancer tissues were obtained with written informed consent, and all experiments were approved by Human Ethics Review Committee (approval no. 1657) and the Committee of Animal Experiments and Gene Recombination (approval no. 26-31) of Wakayama Medical University, Wakayama, Japan.

\section{Patient consent for publication}

Not applicable.

\section{Competing interests}

The authors declare that they have no competing interests.

\section{References}

1. Siegel R, Ma J, Zou Z and Jemal A: Cancer statistics, 2014. CA Cancer J Clin 64: 9-29, 2014.

2. Torre LA, Siegel RL, Ward EM and Jemal A: Global cancer incidence and mortality rates and trends-an update. Cancer Epidemiol Biomarkers Prev 25: 16-27, 2016.

3. Van Cutsem E, Sagaert X, Topal B, Haustermans K and Prenen H: Gastric cancer. Lancet 388: 2654-2664, 2016.

4. Hemminki A, Oksanen M and Merisalo-Soikkeli M: Oncolytic virotherapy trials-letter. Clin Cancer Res 19: 4541-4542, 2013.

5. Russell SJ, Peng KW and Bell JC: Oncolytic virotherapy. Nat Biotechnol 30: 658-670, 2012.

6. Maroun J, Muñoz-Alía M, Ammayappan A, Schulze A, Peng KW and Russell S: Designing and building oncolytic viruses. Future Virol 12: 193-213, 2017.

7. Fukuhara H, Ino Y and Todo T: Oncolytic virus therapy: A new era of cancer treatment at dawn. Cancer Sci 107: 1373-1379, 2016.

8. Mineta T, Rabkin SD, Yazaki T, Hunter WD and Martuza RL: Attenuated multi-mutated herpes simplex virus-1 for the treatment of malignant gliomas. Nat Med 1: 938-943, 1995.

9. Delwar ZM, Liu G, Kuo Y, Lee C, Bu L, Rennie PS and Jia WW: Tumour-specific triple-regulated oncolytic herpes virus to target glioma. Oncotarget 7: 28658-28669, 2016.

10. Longo SL, Griffith C, Glass A, Shillitoe EJ and Post DE: Development of an oncolytic herpes simplex virus using a tumor-specific HIF-responsive promoter. Cancer Gene Ther 18: 123-134, 2011 .

11. Lee CY, Bu LX, Rennie PS and Jia WW: An HSV-1 amplicon system for prostate-specific expression of ICP4 to complement oncolytic viral replication for in vitro and in vivo treatment of prostate cancer cells. Cancer Gene Ther 14: 652-660, 2007. 
12. Streby KA, Geller JI, Currier MA, Warren PS, Racadio JM, Towbin AJ, Vaughan MR, Triplet M, Ott-Napier K, Dishman DJ, et al: Intratumoral injection of HSV1716, an oncolytic herpes virus, is safe and shows evidence of immune response and viral replication in young cancer patients. Clin Cancer Res 23: 3566-3574, 2017.

13. Markert JM, Razdan SN, Kuo HC, Cantor A, Knoll A, Karrasch M, Nabors LB, Markiewicz M, Agee BS Coleman JM, et al: A phase 1 trial of oncolytic HSV-1, G207, given in combination with radiation for recurrent GBM demonstrates safety and radiographic responses. Mol Ther 22: 1048-1055, 2014.

14. Geevarghese SK, Geller DA, de Haan HA, Hörer M, Knoll AE, Mescheder A, Nemunaitis J, Reid TR, Sze DY, Tanabe KK and Tawfik H: Phase I/II study of oncolytic herpes simplex virus NV1020 in patients with extensively pretreated refractory colorectal cancer metastatic to the liver. Hum Gene Ther 21 $1119-1128,2010$.

15. Andtbacka RH, Kaufman HL, Collichio F, Amatruda T, Senzer N, Chesney J, Delman KA, Spitler LE, Puzanov I, Agarwala SS, et al: Talimogene laherparepvec improves durable response rate in patients with advanced melanoma. J Clin Oncol 33: 2780-2788, 2015.

16. Deguchi T, Shikano T, Kasuya H, Nawa A, Fujiwara S, Takeda S, Gewen T, Sahin TT, Yamada S, Kanzaki A, et al: Combination of the tumor angiogenesis inhibitor bevacizumab and intratumoral oncolytic herpes virus injections as a treatment strategy for human gastric cancers. Hepatogastroenterology 59: 1844-1850, 2012.

17. Wong J, Kelly K, Mittra A, Gonzalez SJ, Song KY, Simpson G, Coffin R and Fong Y: A third-generation herpesvirus is effective against gastroesophageal cancer. J Surg Res 163: 214-220, 2010 .

18. Sugawara K, Iwai M, Yajima S, Tanaka M, Yanagihara K, Seto Y and Todo T: Efficacy of a Third-generation oncolytic herpes virus G47 $\Delta$ in advanced stage models of human gastric cancer. Mol Ther Oncolytics 17: 205-215, 2020.

19. Tsuji T, Nakamori M, Iwahashi M, Nakamura M, Ojima T, Iida T, Katsuda M, Hayata K, Ino Y, Todo T and Yamaue H: An arme oncolytic herpes simplex virus expressing thrombospondin-1 has an enhanced in vivo antitumor effect against human gastric cancer. Int J Cancer 132: 485-494, 2013

20. Liu L, Wu W, Zhu G, Liu L, Guan G, Li X, Jin N and Chi B: Therapeutic efficacy of an hTERT promoter-driven oncolytic adenovirus that expresses apoptin in gastric carcinoma. Int $\mathrm{J} \mathrm{Mol}$ Med 30: 747-754, 2012

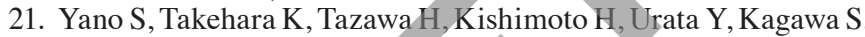
Fujiwara T and Hoffman RM: Therapeutic cell-cycle-decoy efficacy of a telomerase-dependent adenovirus in an orthotopic model of chemotherapy-resistant human stomach carcinomatosis peritonitis visualized with FUCCI imaging. J Cell Biochem 118 3635-3642, 2017

22. Seimiya H: Crossroads of telomere biology and anticancer drug discovery. Cancer Sci 111: 3089-3099, 2020.

23. Zhang C, Chen X, Li L, Zhou Y, Wang C and Hou S: The association between telomere length and cancer prognosis: Evidence from a meta-analysis. PLoS One 10: e0133174, 2015.

24. Watson JD: Origin of concatemeric T7 DNA. Nat New Biol 239 197-201, 1972

25. Hiyama E, Gollahon L, Kataoka T, Kuroi K, Yokoyama T, Gazdar AF, Hiyama K, Piatyszek MA and Shay JW: Telomerase activity in human breast tumors. J Natl Cancer Inst 88: 116-122, 1996.

26. Kim NW, Piatyszek MA, Prowse KR, Harley CB, West MD, Ho PL, Coviello GM, Wright WE, Weinrich SL and Shay JW: Specific association of human telomerase activity with immortal cells and cancer. Science 266: 2011-2015, 1994.

27. Kyo S, Kanaya T, Ishikawa $\mathrm{H}$, Ueno $\mathrm{H}$ and Inoue $\mathrm{M}$ : Telomerase activity in gynecological tumors. Clin Cancer Res 2: 2023-2028, 1996.

28. Sobin LH, Gospodarowicz MK and Wittekind C (eds): TNM Classification of Malignant tumours. 7th edition. Wiley-Blackwell, Hoboken, NJ, USA, 2011.

29. Lü MH, Deng JQ, Cao YL, Fang DC, Zhang Y and Yang SM: Prognostic role of telomerase activity in gastric adenocarcinoma: A meta-analysis. Exp Ther Med 3: 728-734, 2012.

30. Campagna D, Cope L, Lakkur SS, Henderson C, Laheru D and Iacobuzio-Donahue CA: Gene expression profiles associated with advanced pancreatic cancer. Int J Clin Exp Pathol 1: 32-43, 2008 .
31. Meinhold-Heerlein I, Stenner-Liewen F, Liewen H, Kitada S, Krajewska M, Krajewski S, Zapata JM, Monks A, Scudiero DA, Bauknecht T and Reed JC: Expression and potential role of Fas-associated phosphatase-1 in ovarian cancer. Am J Pathol 158: 1335-1344, 2001.

32. Seethala RR, Gooding WE, Handler PN, Collins B, Zhang Q, Siegfried JM and Grandis JR: Immunohistochemical analysis of phosphotyrosine signal transducer and activator of transcription 3 and epidermal grow th factor receptor autocrine signaling pathways in head and neck cancers and metastatic lymph nodes. Clin Cancer Res 14: 1303-1309, 2008.

33. Shimizu A, Hirono S, Tani M, Kawai M, Okada K, Miyazawa M, Kitahata Y, Nakamura Y, Noda T, Yokoyama S and Yamaue H: Coexpression of MUC16 and mesothelin is related to the invasion process in pancreatic ductal adenocarcinoma. Cancer Sci 103: 739-746, 2012

34. Campbell EJ, McDuff E, Tatarov O, Tovey S, Brunton V, Cooke TG and Edwards J: Phosphorylated c-Src in the nucleus is associated with improved patient outcome in ER-positive breast cancer. Br J Cancer 99: 1769-1774, 2008.

35. Cappia S, Righi L, Mirabelli D, Ceppi P, Bacillo E, Ardissone F, Molinaro L, Scagliotti GV and Papotti M: Prognostic role of osteopontin expression in malignant pleural mesothelioma. Am J Clin Pathol 130: 58-64, 2008.

36. Todo T, Martuza RL, Rabkin SD and Johnson PA: Oncolytic herpes simplex virus vector with enhanced MHC class I presentation and tumor cell killing. Proc Natl Acad Sci USA 98: 6396-6401, 2001

37. Fu X, Nakamori M, Tao L, Amato R and Zhang X: Antitumor effects of two newly constructed oncolytic herpes simplex viruses against renal cell carcinoma. Int J Oncol 30: 1561-1567, 2007.

38. Morikawa T, Sugiyama A, Kume H, Ota S, Kashima T, Tomita K, Kitamura T, Kodama T, Fukayama $M$ and Aburatani $\mathrm{H}$ : Identification of Toll-like receptor 3 as a potential therapeutic target in clear cell renal cell carcinoma. Clin Cancer Res 13: 5703-5709, 2007.

39. Morikawa T, Hino R, Uozaki H, Maeda D, Ushiku T, Shinozaki A, Sakatani T and Fukayama M: Expression of ribonucleotide reductase M2 subunit in gastric cancer and effects of RRM2 inhibition in vitro. Hum Pathol 41: 1742-1748, 2010.

40. Passer BJ, Wu CL, Wu S, Rabkin SD and Martuza RL: Analysis of genetically engineered oncolytic herpes simplex viruses in human prostate cancer organotypic cultures. Gene Ther 16: 1477-1482, 2009

41. Nordlund P and Reichard P: Ribonucleotide reductases. Annu Rev Biochem 75: 681-706, 2006.

42. Hsieh YY, Chou CJ, Lo HL and Yang PM: Repositioning of a cyclin-dependent kinase inhibitor GW8510 as a ribonucleotide reductase M2 inhibitor to treat human colorectal cancer. Cell Death Discov 2: 16027, 2016.

43. Eriksson S and Martin DW Jr: Ribonucleotide reductase in cultured mouse lymphoma cells. Cell cycle-dependent variation in the activity of subunit protein M2. J Biol Chem 256: 9436-9440, 1981.

44. Chen $\mathrm{CH}$ and Chen RJ: Prevalence of telomerase activity in human cancer. J Formos Med Assoc 110: 275-289, 2011.

45. Liu Z, Li Q, Li K, Chen L, Li W, Hou M, Liu T, Yang J, Lindvall C, Björkholm M, et al: Telomerase reverse transcriptase promotes epithelial-mesenchymal transition and stem cell-like traits in cancer cells. Oncogene 32: 4203-4213, 2013.

46. Ruden $\mathrm{M}$ and Puri N: Novel anticancer therapeutics targeting telomerase. Cancer Treat Rev 39: 444-456, 2013.

47. Yoo J, Park SY, Kang SJ, Kim BK, Shim SI and Kang CS: Expression of telomerase activity, human telomerase RNA, and telomerase reverse transcriptase in gastric adenocarcinomas. Mod Pathol 16: 700-707, 2003.

48. Yano S, Tazawa H, Hashimoto Y, Shirakawa Y, Kuroda S, Nishizaki M, Kishimoto H, Uno F, Nagasaka T, Urata Y, et al: A genetically engineered oncolytic adenovirus decoys and lethally traps quiescent cancer stem-like cells in S/G2/M phases. Clin Cancer Res 19: 6495-6505, 2013.

49. Watanabe M, Kagawa S, Kuwada K, Hashimoto Y, Shigeyasu K, Ishida M, Sakamoto S, Ito A, Kikuchi S, Kuroda S, et al: Integrated fluorescent cytology with nano-biologics in peritoneally disseminated gastric cancer. Cancer Sci 109: 3263-3271, 2018.

50. Ishikawa W, Kikuchi S, Ogawa T, Tabuchi M, Tazawa H, Kuroda S, Noma K, Nishizaki M, Kagawa S, Urata Y and Fujiwara T: Boosting replication and penetration of oncolytic adenovirus by paclitaxel eradicate peritoneal metastasis of gastric cancer. Mol Ther Oncolytics 18: 262-271, 2020. 
51. Nakatake R, Kaibori M, Nakamura Y, Tanaka Y, Matushima H, Okumura T,Murakami T,Ino Y,Todo T and Kon M:Third-generation oncolytic herpes simplex virus inhibits the growth of liver tumors in mice. Cancer Sci 109: 600-610, 2018.

52. Matsushima H, Kaibori M, Hatta M, Ishizaki M, Nakatake R, Okumura T, Yoshii K and Todo T: Efficacy of a third-generation oncolytic herpes simplex virus in neuroendocrine tumor xenograft models. Oncotarget 10: 7132-7141, 2019.

53. Lam JT, Kanerva A, Bauerschmitz GJ, Takayama K, Suzuki K, Yamamoto M, Bhoola SM, Liu B, Wang M, Barnes MN, et al: Inter-patient variation in efficacy of five oncolytic adenovirus candidates for ovarian cancer therapy. J Gene Med 6: 1333-1342, 2004.
54. Ribas A, Dummer R, Puzanov I, VanderWalde A, Andtbacka RHI, Michielin O, Olszanski AJ, Malvehy J, Cebon J, Fernandez E, et al: Oncolytic virotherapy promotes intratumoral $\mathrm{T}$ cell infiltration and improves Anti-PD-1 immunotherapy. Cell 174: 1031-1032, 2018.

(i) $\odot$ This work is licensed under a Creative Commons Attribution-NonCommercial-NoDerivatives 4.0 International (CC BY-NC-ND 4.0) License. 